\title{
Statt eines Schlussworts - 12 Merksätze für konstruktive Gespräche
}

1. Gespräche bilden die Nervenbahnen einer Organisation.

2. Die Gesprächsteilnehmer sind jeweils in drei Bezugswelten zuhause, die nicht völlig deckungsgleich sind: in ihrer Privatwelt, in ihrer Fachwelt und in der Organisationswelt. Je größer der gemeinsame Bezugsrahmen der Teilnehmer (ihr Common Ground) ist, desto besser sind die Chancen für eine effektive Verständigung.

3. Zielorientierung ist für Gespräche im beruflichen Umfeld kennzeichnend. Die Fokussierung auf das eigene Ziel macht Zuhören zur Schwerstarbeit.

4. Jedes Gespräch lebt von den gegenseitigen Annahmen der Gesprächsteilnehmer. Ein konstruktives Gespräch entwickelt sich durch dynamisches Nachjustieren der Annahmen über das Gegenüber. Die jeweiligen Antworten signalisieren, in welche Richtung nachjustiert werden muss.

5. Eine gute Vorbereitung fließt in ein Gespräch ein, bestimmt aber nicht allein dessen Verlauf.

6. Eine ausbalancierte Statuswippe achtet das Bedürfnis des Gegenübers nach Wertschätzung.

7. Informationsfluss lässt sich durch Nachfragen sicherstellen. Lieber nachfragen als den Macher spielen, der alles weiß - das schafft mehr Optionen für eine konstruktive Lösung.

8. Wirksames Argumentieren fängt mit dem Zuhören an. Machen Sie sich klar, was Sie bei Ihrem Gegenüber erreichen wollen.

9. Bleiben Sie dran: Wirksame Argumente brauchen manchmal einen langen Atem.

10. Der besonnene Umgang mit Emotionen bildet die Basis eines gelungenen Gesprächs. Emotionen lassen sich nicht wegrationalisieren. Gefühls- und Denkverbote verhindern eine fachlich angemessene Lösung.

11. Wenn Bedürfnisse aus der Ich-Perspektive formuliert werden, muss sich das Gegenüber nicht angegriffen fühlen.

12. Neue Verhaltensweisen müssen trainiert werden - so wie Muskeln. 